\title{
Asymptomatic hypoglycemia among children with acute lymphoblastic leukemia on maintenance therapy
}

\author{
Janan G. Hassan ${ }^{\star, \dagger, 1}$, Ayad .A. Mohamed ${ }^{2}$ \\ ${ }^{1}$ CABP. Prof. in pediatric / Department of pediatrics Medical College. Basra University Basra maternity and child teaching hospital \\ ${ }^{2}$ M.B.Ch.B/ Basra maternity and child teaching hospital \\ DOI: https://doi.org/10.15520/ijmhs.v9i1.2430
}

Accepted 29 Dec 2018; Received 3 Dec 2018; Publish Online 04 Feb 2019

\author{
Reviewed By: Dr. \\ Daniel V. \\ Department: Medical
}

\begin{abstract}
Background: Knowledge of the adverse effects of maintenance chemotherapy, therapy in children with acute lymphoblastic leukemia being treated according to the MRC modified protocols.

Objective: To figure out the asymptomatic hypoglycemia in a sample of children patients at a stage of maintenance therapy.

Methods: Prospective study was carried out over 6 months from the 1st of January 2004 till the 30th of June 2004. A total sample of 30 patients aged between (1 and 15 years) with acute lymphoblastic leukemia were included in study who were treated at Basra Maternity and child teaching hospital, all of them were being treated according to MRC modified protocol and on maintenance therapy (6 mercaptopurina + methotroxate), 35 healthy children matched for age and sex randomly selected as control.

Results: Hypoglycemia were seen is 18 (60\%) of patients with leukemia, 10 (55.5\%) females and 8 (44.4\%) males. Blood glucose level $<3.33 \mathrm{mmol} / \mathrm{L}$ during 12 hours of overnight fasting.

Conclusion: Hypoglycemia is the most common adverse effect in children with acute lymphoblastic leukemia on maintenance therapy.
\end{abstract}

Key words: asymptomatic hypoglycemia-acute lymphoblastic leukemia.

\section{INTRODUCTION}

Acute leukemia is the most common malignancy in children, accounting for about $25 \%$ of all cancer under 5 years of age [1] , and acute lymphoblastic leukemia remains the most common malignancy in children, it accounts for one fourth of all childhood cancers and approximately $75 \%$ of all cases of childhood leukemia. Hypoglycemia is based on whether symptoms occur in the fasting state or following food intake (post prandial ) [2, 3] . And most common causes of hypoglycemia are:- drugs (insulin, sulfonylurea, pentamidine, sulfonamide, salicylate, 6 mercaptopurina, thioguanine and methotroxate, endogenous hyperinsulinema like insulinoma, autoimmune ( autoantibodies to insulin ) and ectopic insulin secretion, critical illnesses hepatic, renal or cardiac failure, sepsis and starvation, endocrine deficiencies (cortisol, growth hormone, glucagons, epinephrine), non -B

\footnotetext{
${ }^{\star}$ Corresponding author.

$\dagger$ Email: jenan_ah03@yahoo.com.
}

cell tumors (fibrosarcoma, mesotheliome, Rhahdomyosarcoma, hepatoma, adrenocortical tumors, leukemia, lymphoma, melanoma and tetratoma, disorder of infancy or childhood (transient intolerance of fasting infant of diabetic mother, inherited enzyme defect ), postprandial (reactive after gastric surgery, ethanol -induced, autonomic symptoms with true hypoglycemia, factitions (insulins, sulfouylureas. Hgpoglycemia present with symptoms and signs of hypoglycemia when the blood glucose concentrate is about $2-5 \mathrm{mmo} / \mathrm{L}$ and the most common manifestation of hypoglycemia reflect sympathetic adrenergic stimulation of autonomic nervous system and neurological disturbances, autonomic nervous system include pallor, sweating, weaknes , nausea and vomiting, abdominal pain and hunger while neuroglycopuenia sign are confusion, irritability, headach, visual disturbance, unusual behaviour, come and convulsion $[4,5]$.

Most Biochemical changes in children with acute lymphoblastic leukemia and with a tendency to fasting hypoglycemia are one of the complications specially in young, 


\section{Asymptomatic hypoglycemia among children with acute lymphoblastic leukemia on maintenance therapy

thin children who are taking purine analogues such as mercaptopuriue and thioguanine which are similar to finding in idiopathic ketotic hypoglycemia of childhood, characteristics include raised serum level of free fatty acids and ketone bodies, appropriate endocrine responses and normal plasma level of laclate but low level of alanine [5-7]

The relationship between the hypoglycemic event and last meal provide some important help for diagnosis when hypoglycemia event occurs less than 12 hours after meal as impaired glycogenolysis occurring 12 to 16 hours after the meal may be due to impair gluconeogenesis [8] .

So to study whether hypoglycemia is a potential adverse effect after an overnight fast of children with acute lymphoblastic leukemia on maintenance therapy.

\section{PATIENTS AND METHODS}

\section{Patients:}

Prospective study was carried out over 6 months from the first of January 2004 till the end of June 2004.

The study included 30 children on maintenance treatment (MT) who were visited outpatient oncology clinic for follow up or admitted to inpatient for the treatment at Basra Maternity and Child Teaching Hospital ,

All patients with Acute Lymphoblastic Leukemia(ALL) had a comprehensive diagnostic work up for accurate diagnosis' and had been treated according to the modified MRC protocol in which maintenance treatment include Vincristin monthly and oral (6 mercaptopurine daily and Methotroxate weekly).

Fourteen of the patients were girls and sixteen were boys. The median age was 5.5 years at the time of the study. History and physical examination were carried out, and the following information was recorded: date of diagnosis, age, sex, residence, duration of disease, duration of MT.

General examination (including vital signs, measurement like body weight, height ) and systemic examination of the chest, cardiovascular system, abdominal, central nervous system.

\section{Control group:}

Thirty five children (18 male and 17 female) who were admitted to Basra maternity and child Teaching hospital for chest infection and diarrheal disease after stabilization of their condition, critically ill children were excluded from the study, were randomly selected as control, matched for age and sex and the following in formations were recorded: age, sex, residence, vital signs, body weight, height or length and systemic examinations.

\section{Methods:}

To study fasting tolerance an overnight fasting test of up to 12 hours was performed on each study patients in oncology unit of Basra Maternity and Child Hospital. Fasting was routinely started at 8 p.m. following normal daily food intake and normal meals. Blood samples for immediate measurement of blood glucose level were taken before starting MT and after a period of MT as maintance treatment was started at 39 week of therapy, the test was done between 50 to 100 week. Fasting hypoglycemia was defined as a blood glucose level $<3.3 \mathrm{mmol} / \mathrm{L}^{(3)}$.

Immediate blood glucose levels were assessed using glucometer (glucotrend 2 is trade mark of a member of the roche group. UK) code number 765 .

\section{Statistical analysis:}

1. Statistical analysis was done to find the P-value using SPSS version 11.0 computer program

- The results of clinical and biochemical variables were expressed as mean and standard deviation of the mean $(\mathrm{M}+\mathrm{SD})$ and as numbers and percentages: $\mathrm{n}(\%)$.

- Chi-square (X) test was carried out to determine the relative important of different variables.

- T-test was carried out to compare between sample proportions.

- Logistic regression analysis was carried out to determine the importance of different variables.

1. P-value $>0.05$ considered to be not significant (NS).

\section{$3 \quad$ RESULTS}

Table 1. Characteristics of the studied groups

\begin{tabular}{lllll}
\hline Parameters & & $\begin{array}{l}\text { Patients } \\
\mathrm{N}=30\end{array}$ & $\begin{array}{l}\text { Control } \\
\mathrm{N}=35\end{array}$ & P-value \\
$\begin{array}{l}\text { Age } \\
\text { (year)* }\end{array}$ & & $6.31 \pm 2.89$ & $6.48 \pm 4.5$ & 0.614 \\
Sex & Male & $16(53.3 \%)$ & $18(51.4 \%)$ & 0.538 \\
& Female & $14(46.7 \%)$ & $17(48.6 \%)$ & 0.878 \\
Residence & Urban & $8(26.7 \%)$ & $15(42.9 \%)$ & 0.202 \\
BMI & Rural & $22(73.3 \%)$ & $20(57.1 \%)$ & 0.133 \\
\hline
\end{tabular}

* Values were expressed as mean $\pm \mathrm{SD}$

$\mathrm{NS}=\mathrm{P}>0.05, \mathrm{~S}=\mathrm{P}<0.05, \mathrm{HS}=\mathrm{P}<0.01$

Basic characteristics of the studied groups:

A total of 65 children aged ranged from (1.5-15 years) and the mean 6.3 were included in the study; 30 of them were with ALL. The characteristics of the studied children (patients and controls) are illustrated in Table 1 .

This table shows that there was no significant statistical difference in the mean age, sex, residence and BMI between patients with ALL and the control group.

characteristics of the patients with ALL who were received MT consist of weekly oral MTX and daily $6 \mathrm{MP}$ were evaluated in regard to fasting blood glucose classified as hypoglycemia $\leq 3.33 \mathrm{mmol} / \mathrm{L}$ and normoglycemia $>3.33 \mathrm{mmol} / \mathrm{L}$.

Eighteen out of 30 patients (60\%) develop hypoglycemia after 12 hours of overnight fasting, 7 out of 18 had blood glucose levels below $2.5 \mathrm{mmol} / \mathrm{L}$ Table 2 .

This Table 3 shows glucose level in relation to age group,(11) out of (18) with hypoglycemia $(61.1 \%)$ were below 6 years of age. Only (5) out of the (12)children (41.6\%) 
Table 2. characteristics of the patients with ALL in relation to glucuose level

\begin{tabular}{|c|c|c|c|c|}
\hline Parameter & & $\begin{array}{l}\text { Normoglycemia } \\
\mathrm{n}=12\end{array}$ & $\begin{array}{l}\text { Hypoglycemia } \\
\mathrm{n}=18\end{array}$ & P-value \\
\hline $\begin{array}{l}\text { Age } \\
\text { (year)* }\end{array}$ & & $6.5(4-10)$ & $5.5(1.5-14)$ & \\
\hline & Male & $8(66.6 \%)$ & $8(44.4 \%)$ & \\
\hline Sex & Female & $4(33.3)$ & $10(55.5 \%)$ & \\
\hline & Urban & $3(25 \%)$ & $7(38.2 \%)$ & \\
\hline Residence & Rural & $9(75 \%)$ & $11(61.1 \%)$ & \\
\hline BMI & & $23.95 \pm 3.12$ & $23.45 \pm 2.13$ & \\
\hline $\begin{array}{l}\text { Duration } \\
\text { of dis- } \\
\text { ease/month }\end{array}$ & & $32.87 \pm 17.25$ & $17.5 \pm 15.05$ & \\
\hline $\begin{array}{l}\text { Duration } \\
\text { of }\end{array}$ & & $8.18 \pm 5.75$ & $5.16 \pm 3.12$ & \\
\hline MT/month & & & & \\
\hline $\begin{array}{l}\text { Dose of } \\
\text { MTX }\end{array}$ & & $14.61 \pm 2.27$ & $13.6 \pm 2.87$ & \\
\hline $\begin{array}{l}\text { Dose of } \\
6 \mathrm{MP}\end{array}$ & & $54.81 \pm 8.53$ & $51.1 \pm 10.7$ & \\
\hline FBS & & $5.8 \pm 1.25$ & $2.45 \pm 0.31$ & HS \\
\hline
\end{tabular}

*Results were expressed as mean $\pm \mathrm{SD} \mathrm{S}=\mathrm{P}<0.05, \mathrm{HS}=\mathrm{P}<0.01$

Table 3. Fasting blood glucose concentration in children $\leq 6$ years and $>6$ years of the age.

\begin{tabular}{llll}
\hline Age & $\begin{array}{l}\text { Hypoglycemia } \\
\text { groupn=18 }\end{array}$ & $\begin{array}{l}\text { Normoglycemia } \\
\text { group } \\
\mathrm{n}=12\end{array}$ & $\begin{array}{l}\text { P- } \\
\text { value }\end{array}$ \\
$\begin{array}{l}56 \\
\begin{array}{l}\text { years } \\
>6\end{array}\end{array}$ & $11(61.1 \%)$ & $5(41.6 \%)$ & \\
years & $7(38.9 \%)$ & $7(58.4 \%)$ & \\
\hline
\end{tabular}

$* \mathrm{~S}=\mathrm{P}<0.05, \mathrm{NS}=\mathrm{P}>0.05$

Table 4. Fasting glucose level before and during MT in patients with ALL

\begin{tabular}{|c|c|c|c|}
\hline $\begin{array}{l}\text { Parame- } \\
\text { ter }\end{array}$ & Before $\mathrm{MTn}=30$ & $\begin{array}{l}\text { During MT } \\
\mathrm{n}=30\end{array}$ & $\begin{array}{l}\mathrm{P}- \\
\text { value }\end{array}$ \\
\hline FBS & $5.88 \pm 1.29$ & $3.71 \pm 1.63$ & $<0.001$ \\
\hline
\end{tabular}

* Values were expressed as mean \pm SD P $<0.001=\mathrm{HS}$

who were under the age of 6 years had normal blood glucose level during fasting

Table 4 shows that there was highly significant low glucose level during MT as compare before MT.

Table 5. Characteristics feature of patients with hypoglycemia and control group

\begin{tabular}{|c|c|c|c|c|}
\hline \multicolumn{2}{|l|}{ Parameters } & Patients & Control & \multirow[t]{2}{*}{$\mathrm{P}$-value } \\
\hline & & $\mathrm{N}=18$ & $\mathrm{~N}=35$ & \\
\hline Age & & $6.12 \pm 3.5$ & $6.48 \pm 4.5$ & 0.02 \\
\hline \multirow{2}{*}{ Sex } & Male & $8(44.4 \%)$ & $18(51.4 \%)$ & \multirow{2}{*}{0.252} \\
\hline & Female & $10(55.5 \%)$ & $17(48.6 \%)$ & \\
\hline \multirow{2}{*}{ Residence } & Urban & $5(27.8 \%)$ & $15(42.9 \%)$ & \multirow{2}{*}{0.375} \\
\hline & Rural & $13(72.2 \%)$ & $20(57.1 \%)$ & \\
\hline BMI & & $23.54 \pm 2.17$ & $28.8 \pm 2.84$ & 0.312 \\
\hline FBS & & $2.45 \pm 0.31$ & $5.8 \pm 1.29$ & 0.001 \\
\hline
\end{tabular}

* Results were expressed as mean $\pm \mathrm{SD}$
Table (5) shows that there was low fasting glucose level in patients as compare to control group and stastisticaly highly significant but there was no significant difference between hypoglycemic group and control group in respect of sex , residence and BM . Moreover, there was significant differences between hypoglycemic group and control group in respect of age.

Table 6. Relations between patients on MT and hypoglycemia after adjustment to $F B S$ and residence.

\begin{tabular}{lll}
\hline Variables & S.E & Significance \\
FBS & 0.286 & 0.001 \\
Residence & 0.72 & 0.05
\end{tabular}

Table 6 shows logistic regression analysis to study the relation between MT of the patients with ALL and hypoglycemia after adjustment for FBS and residence, it illustrates the followings:

There was positive association between MT of the patients with ALL and hypoglycemia with standard error 0.286 . There was positive association between residence of the patients on MT and hypoglycemia with standard error 0.72. After adjustment for FBS, the relation between MT of patients with ALL and hypoglycemia still highly significant $(\mathrm{P}=0.001)$. After adjustment for residence of patients on MT, there was also significant association between MT of patient with ALL and hypoglycemia $(\mathrm{P}=0.05)$.

Table 7. Hypoglycemic

\begin{tabular}{lll}
\hline Parameters & Symptom & No symptom \\
Hypoglycemic group & $3(16.6 \%)$ & 18 \\
\hline
\end{tabular}

Only (3) patients out of 18 exhibited one symptom of hypoglycemia (2) patients were drowsy or restless and only one patient develop sweating as shown in Table 7 


\section{Asymptomatic hypoglycemia among children with acute lymphoblastic leukemia on maintenance therapy

\section{DISCUSSION}

Acute lymphoblastic leukemia is one of leading causes of mortality and morbidity worldwide, with increasing numbers of children cured of leukemia there has been an increased awareness of the adverse effects and late sequale of therapy.

Our study showed that fasting hypoglycemia is a potential adverse effect of MT in children being treated according to the MRC modified protocol.

Eighteen out of 30 patients (60\%) develop hypoglycemia after 1\%)2\%) hours of overnight fasting. Similar results was observed by Halonen et al [9] . that was fasting hypoglycemia was common during maintenance therapy for childhood ALL. In their study of 35 consecutive patients on MT, 16hrs overnight fasting was followed by hypoglycemia in 19 children (54\%).

Children under six years of age are especially prone to fasting hypoglycemia, in our study $(61.1 \%)$ of the children with hypoglycemia were younger than 6 years of age. Moreover $(41.6 \%)$ of the children under the age of six years remained normoglycemic during fasting, two third of all study patients developed hypoglycemia within 12 hours of fasting. This is a pathological finding, since the normal lower limit for a blood glucose level in healthy children aged 3 to 15 years after a 12-hours fast is reported to be $3.7 \mathrm{mmol} / \mathrm{L} \mathrm{[10]}$ .This agree by similar study was observe by Makipernaa A. et. al. [11]

This indicates children with ALL in the course of overnight sleep are at risk of becoming hypoglycemia, with or without symptoms. No differences in sex, BMI, or residence between the patients with hypoglycemia and those with normoglycemia were detected, and did not explain the tendency to hypoglycemia. [9] Surprisingly little is known about overnight blood glucose levels in normal children. Cyclical variation, periodicity 80-120 min., is described with a gradual fall until wakening with no evidence of a dawn blood glucose rise $[12,13]$.

In some children levels fall to $<3 \mathrm{mmol} / \mathrm{L}$. Maintenance of normoglycemia seems largely mediated through free fatty acid metabolism (with significant differences between 8p.m and 8a.m B hydroxybutyrate level) whereas lactate levels suggest that glycogen stores are relatively protected overnight but available for acute hypoglycemia crises [13]

In children with idiopathic ketotic hypoglycemia the glycemic response to glucagon has also been reported to be less after fasting, suggesting that hepatic glycogen is more rapidly exhausted in them [14] .

Young children tolerate fasting less well than adults, blood glucose levels may start to fall after periods as short as 6-12 hours, reason are unclear [15] .

Glucose levels appear to fall in a cyclical fashion overnight in normal children, some falling into the hypoglycemic range before compensation [16] .

The impaired fasting tolerance was obviously associated with the MT, particularly with the $6 \mathrm{MP}$ and MTX. There were no significant differences between the hypoglycemic and normoglycemic children with ALL in relation to the dose levels of $6 \mathrm{MP}$ and MTX.

This suggests that concentrations or cumulative doses of 6MP and MTX do not need to reach particular levels for hypoglycemia to occur ${ }^{[17]}$. The question which also remained unanswered in our study is whether repeated highdose MTX courses included in the MRC modified protocol MT of ALL are associated with the tendency to hypoglycemia. No reports concerning this issue are available with different therapy regimens. In our study only 3 patients out of 18 exhibited one symptom of hypoglycemia $16.6 \%$, this results also was observed by recent retrospective Italian study report symptomatic hypoglycemia in 6 out of 86 children $(6.9 \%)$ receiving oral purine analogues for treatment of childhood ALL [18] .

Symptomatic hypoglycemia episodes occurred throughout the entire treatment period except for the induction phase: consolidation phase, reinduction phase, and continuation phase, the researcher also suggest that the administration of the purine analogues 6MP and 6TG seems to be associated with this event, more than the combination of $6 \mathrm{MP}$ and MTX [18] .

It is widely known that liver damage, abnormal liver tests, and abnormal liver biopsy results are among findings during oral 6MP and MTX therapy [17].

Both 6MP and MTX are anti-metabolites, $6 \mathrm{MP}$ is a purine analogue and MTX is a folic acid analogue. They affect the synthesis of RNA and DNA precursors and the functioning of normal cells .

Therapy related fasting hypoglycemia during overnight is often hazardous and insidious. Although not threatening in our experience, it was very frightening for the parents, who developed anxiety and sometime even sleep disturbances.

The mechanisms by which such oral antimetabolite therapy may elicit symptomatic hypoglycemia or asymptomatic in children under chemotherapy for ALL remain unclear. Impaired turnover of glycogen can be hypothesized, but not recommended at present; alteration of liver enzyme is for more common, additional predisposing factors are associated with reduced fat storage and with young age as risk factor ${ }^{[18]}$

As hypoglycemia was significantly adverse effect in MT of the patients so children under the age of 6 years and thin build child should be prescribe shorter fasting intervals, with late evening meal rich in complex carbohydrates and protein with early breakfast. Further studies are required to identify the mechanism by which hypoglycemia develop in children with ALL on maintance therapy.

\section{REFERENCES}

[1] Hay WW, MD ARH, Levin MJ, Sondheimer JM. MD.A lange medical book, current pediatric diagnosis and treatment, Appleton and Lange; 1999.

[2] Cecil,essentials of medicine. W.B.Saunders Company; 1993.

[3] Wyngaarden JB, Smith LH Jr., Bennet JC (eds.):Service FJ. Hypoglycemic disorder. In: Cecil Textbook of Medicine. Philadelphia: W.B.Saunders Co; 1992.

[4] Principles of internal medicine. vol. 1998; 
[5] Clinical paediatric endocrinology. Oxford: Ed.Brook COD, Blackwell Science Ltd; 1995. p. 677-693.

[6] F W, MD Z, Jack, editors. and Deloris. Review of Medical Physiology. of; 1983.

[7] Chaussain JL. Glycemic response to $24 \mathrm{hr}$ fast in normal children and children with ketotic hypoglycemia. J Paediatric;1978(82):438.

[8] A FP, R P. Morgese G and Chiarelli F. Hypoglycemia in childhood: A clinical approach. J Pediatric Endocrinology Metabolic. 1998;11(11):147-152. 8] Verrotti.

[9] P SMK, A M. Department of paediatrics, tampere university Hospital. Tampere, Finland; 2000. Available from: www. ncbi.n1m.nih.gov/entrez/anery.com.

[10] Lamers KJ, Doesburgh WH, Gabreels FJ, Romson AC, Renier WO, Wevers RA and Lemmens WA. Reference values of blood components related to fule metabolism in children after an overnight fast. Clin Chim Acta;1985(145):17-26.

[11] Makipernaa,et.al. Paediatric Research Center,Medical School, University of Tampere,Finland.2003.www.paivi.halonen.@ kotiposti.net Forfar and Arneil's textbook of pediatric edited by A.G.M.Campbell, Professor of Child Heath, University of Aberdeen, Aberdeen, UK, Neil McIntosh, Professor of Child Life and Health, University of. Edinburgh, Edinburgh: UK; 1998.

[12] Stirling HF, Darling JAB, Kelnar CJH. Nocturnal glucose homeostasis in normal children. Hormone Research. 1991;35: 54 (abstract 210.

[13] JL GP, JC OGJ. Glycemic response to 24 hour fast in normal children and children with ketotic hypoglycemia: II. Hormonal and metabolic changes. JPediatr;1976(85):776781.

[14] Kelnar CJH. Hypoglycemia in children undergoing adentonsillectomy. British Medical Journal. 1976;p. 751-752.

[15] HF BDGD, CJH K. A familial growth hormone releasing factor deficiency in pseudohypo-parathyrodism. Archives of Disease in Childhood. vol. 66; 1991.

[16] M MD, A Z, VS B, Z V, E G, EF S, et al. Hepatotoxicity of 6 mercapto purine in childhood acute lymphoblastic leukemia: pharmacokinetic characteristics. Med Pediatric Oncol;1996(26):85-89.

[17] Ziino. Symptomatic hypoglycemia in children receiving oral purine analogues for treatment of childhood acute lymphoblastic leukemia. Med Pediatr Oncol;2002(39):32-34.

[18] Perters GJ, Schornagel JH and Milano GA. Clinical pharmacokinetics of anti-metabolites. Cancer Surv;1993(17):123156.

\section{AUTHOR BIOGRAPHY}

Janan G. Hassan CABP. Prof. in pediatric / Department of pediatrics Medical College. Basra University Basra maternity and child teaching hospital

Ayad .A. Mohamed M.B.Ch.B/ Basra maternity and child teaching hospital 